\title{
Sudden Infant Death Syndrome: Forensic Autopsy Findings in Post-neonatal Deaths. Bogota, Colombia 2010
}

\author{
María Luisa Latorre ${ }^{1^{*}}$, Carolina Zambrano ${ }^{2}$ and Sandra Moreno ${ }^{3}$
}

${ }^{1}$ Public Health, Universidad de los Andes, Bogotá, Colombia, USA

${ }^{2}$ Universidad de los Andes, Bogotá, Colombia, USA

${ }^{3}$ Instituto Nacional de Medicina Legal y Ciencias Forenses, Bogotá, Colombia, USA

*Corresponding author: María Luisa Latorre, Researcher Universidad Nacional de Colombia, Calle 127C\#78 A-32 Apto 213, Bogota D.C., Colombia, USA, Tel: 573102721841; E-mail: ml.latorre75@uniandes.edu.co

Received date: February 09, 2016; Accepted date: April 21, 2016; Published date: April 27, 2016

Copyright: $@ 2016$ Latorre ML, et al. This is an open-access article distributed under the terms of the Creative Commons Attribution License, which permits unrestricted use, distribution, and reproduction in any medium, provided the original author and source are credited.

\begin{abstract}
Background: The Sudden Infant Death Syndrome (SIDS) is diagnosed by default when it is not possible to identify the cause of infant death after a complete study including forensic autopsy and death scene investigation. However, Colombia does not mandate a complete study of these deaths, and only some of these cases meet that standard. This research will show the results of those forensic autopsy performed of post neonatal infants deaths in Bogota', Colombia, in 2010, to call attention of the national sanitary authority to the importance of conducting a comprehensive study to conclude what caused the death of these children, who, without it, generally remain reported as "under study" or "indeterminate".
\end{abstract}

Objective: To describe the findings in the forensic autopsy performed in post neonatal subjects at the National Institute of Legal Medicine and Forensic Sciences (INMLCF) in Bogotá, Colombia, in 2010, in order to characterize and describe what could be compatible with SIDS or other sudden and unexpected infant deaths (SUID) meeting international standards.

Methods: The information collected by the INMLCF of all post neonatal deaths that were the subject of medicolegal autopsy was reviewed; the infant case population was characterized by age, sex, cause and manner of death, and the other variables available for study, including the known risk factors for SIDS.

Results: We studied 150 cases of neonatal and post neonatal deaths with medico legal autopsy. In 36 cases the cause of death was determined including two cases of stillbirth. The remaining 114 cases were reviewed and characterized individually, using all available information; 95 deaths were considered compatible with SIDS/SUDI.

Conclusions: It is necessary to have a standard protocol to study infant deaths in Colombia to quantify the SIDS/ SUDI problem. However, with the available information it is possible to estimate a baseline that reflects the magnitude of the problem and contribute to its solution.

Keywords: Sudden infant death syndrome; Sudden and unexpected infant deaths; Autopsy; Forensic autopsy; Postneonatal mortality

\section{Introduction}

The Sudden Infant Death Syndrome (SIDS) is part of the class of sudden and unexpected infant deaths (SUID). SIDS is the cause of death assigned to infant deaths (usually children under 1 year), most often during sleep, which cannot be explained after full investigation of the case including scene investigation, forensic autopsy and review of the infant and family medical history, and are coded according to the WHO Tenth Revision of the International Classification of Diseases ICD-10 - as R95.

Other sudden and unexpected infant deaths (SUID), especially those related to sleep, after investigation, can be attributed to choking, suffocation, trauma, respiratory infections, metabolic disorders or infanticide [1]. Finally, there is a group of sudden and unexpected deaths in which there is not a full investigation, and when no cause is found they are coded according to ICD 10 as R98 and R99.
There are a group of events that occur in infants, mostly under 1 year of age, suddenly and unexpectedly; some are inexplicable, others may have some explanation as accidental suffocation and others have unknown causes. There are protocols for the study of SIDS [2,3] but Colombia does not presently have one. One particular problem that cannot be resolved is that when an infant is found moribund or dead, the parents or other caregiver will pick up the infant and attempt resuscitation while calling for emergency medical help. Consequently, the death scene is disturbed and reconstruction of exact positioning is uncertain.

The SIDS cause remains unknown but risk factors are associated with it, such as the prone sleeping position, 0 co-sleeping with family members, parental tobacco smoking and alcohol consumption by a cosleeping parent, soft sleeping surfaces, pillows, and hyperthermia [4].

Using vital statistics it has been possible for us to develop an approach to defining the magnitude of the problem in Bogota'. In 2010 it is estimated that the number of SIDS was 114 and $110 \mathrm{had}$ a forensic autopsy. 
Citation: Latorre ML, Zambrano C, Moreno S (2016) Sudden Infant Death Syndrome: Forensic Autopsy Findings in Post-neonatal Deaths.

Page 2 of 5

This makes it essential to review the results of the post mortem studies, in post neonatal populations at the National Institute of Legal Medicine and Forensic Sciences (INMLCF) in Bogotá, Colombia, in 2010, in order to characterize and describe what could be compatible with Sudden Infant Death Syndrome (SIDS) or other sudden and unexpected infant deaths (SUID) as defined elsewhere $[5,6]$.

\section{Methodology}

This study was performed in 2014 at the Legal Medicine and Forensic Sciences National Institute (INMLCF), Bogota', Colombia. This Institute does medico forensic autopsy in Colombia and Bogotá, Capital District, has both the largest number of cases and one of the highest SIDS rates in the country.

It is a descriptive observational cross-sectional study, in a population of children older than 1 month and younger than 1 year old who died in Bogota in 2010 whose deaths were investigated by INMLCF.

Data collection: This information was collected using the survey instruments designed in the previous stage, from database of the Identification and Registry of Missing People and Corpses System (SIRDEC) and, another part of the information was obtained using the autopsy reports. Finally, to get more uniform details in regard to the general characteristics of these deaths of the infants, some dossiers were revised to include these updates $[7,8]$.

Information Systematization and Consolidation: the SIRDEC information system has 39 variables. Among these variables, 21 were chosen allow the identified cases to be used for the study. Attached to these cases were the results of the forensics report. Each of the cases was reviewed and the cases were classified as SIDS or SUDI. Also, the identified risk factors were analyzed.

It is important to note that, according to the Colombian INMLCF's Forensic Autopsy Guide, "the forensic pathologists are to provide their expert opinion" including the manner and cause of death.

The manner of death refers to the circumstances that caused the decease which can be either natural death (caused by an illness), accidental (non-intentional caused by the forces of nature or human beings), suicide (self-inflicted and intentional), homicide (caused intentionally or non-intentionally by a third party), under study (if it requires further investigation) or undetermined (it's not precise to establish the manner of death) [9].

This analysis includes the findings from the SIRDEC's database, the autopsy reports and the complete review of each of the case's files that are compatible with SIDS.

\section{Results}

\section{Study sample characteristics - information obtained in SIRDEC data base}

During 2010 the INMLCF performed 32,843 autopsies in Colombia, among these, 5,136 was performed in Bogota.

According to the SIRDEC, it was reported 150 cases of post neonatal (infants older than 30 days and younger than 365 days) death with autopsies between January 1st and December 31st 2010 in Bogota (Table 1).

\begin{tabular}{|l|l|l|l|}
\hline Colombia & Total Mortality & Autopsy & $\%$ \\
\hline Neonatal mortality (<1 month) & 5,179 & 430 & 8.3 \\
\hline $\begin{array}{l}\text { Post neonatal mortality }(>1 \text { month } \\
\text { and }<1 \text { year) }\end{array}$ & 3,176 & 501 & 15.77 \\
\hline Total infant mortality (<1 year) & 8,355 & 931 & 11.14 \\
\hline Total mortality (all ages) & 200,524 & 32,843 & 16.38 \\
\hline Bogota & Total Mortality & Autopsy & $\%$ \\
\hline Neonatal mortality (<1 month) & 845 & 72 & 8.52 \\
\hline $\begin{array}{l}\text { Post neonatal mortality }(>1 \text { month } \\
\text { and }<1 \text { year) }\end{array}$ & 471 & 150 & 31.85 \\
\hline Total infant mortality (<1 year) & 1,316 & 222 & 16.87 \\
\hline Total mortality (all ages) & 32,857 & 5,136 & 15.63 \\
\hline
\end{tabular}

Table 1: Mortality and autopsies in Colombia and Bogota 2010 (Source: DANE and INMLCF).

Among these 150 post neonatal infants deaths, 86 (57.3\%) were male and $64(42.7 \%)$ were female. All of the children were younger than 1 year old, with a peak of incidence of death between the second and the fourth month of life. The place of death was home in 91 cases (60.6\%), hospital in 22 cases (14.6\%), and other locations in 9 cases (6.0\%) and without information in 28 cases (18.6\%).

The reports of the infant's activity at the time of death show that 39 children died during sleep, 22 children were doing some activity at home, 84 were without any information, and 5 cases were reported as "other."

The manner of death results were: natural death in 72 cases (48\%), in study 58 cases $(38.6 \%)$, indeterminate 8 cases $(5.3 \%)$, violent accidental 3 cases $(2 \%)$, violent homicide 1 case $(0.6 \%)$ and violent indeterminate 8 cases $(5.3 \%)$.

The causes of death, defined as disease or event that triggers the sequence leading to death [9], were not always found in the results of the autopsy. The results were trauma in 8 cases, including 2 cases of suffocation caused by airway obstruction. Other diagnoses were pneumonia (22), congenital malformations (7 cases), and 19 cases of intestinal infection or disease, hepatic causes, meningitis and influenza. 107 cases were reported as "cause of death under study" (Table 2).

\begin{tabular}{|l|l|l|}
\hline Variable & $\mathbf{n}$ & $\%$ \\
\hline Sex & \multicolumn{2}{|l|}{} \\
\hline Male & 86 & 57.3 \\
\hline Female & 64 & 42.7 \\
\hline Place of death & \multicolumn{2}{|l|}{} \\
\hline Home & 91 & 60.6 \\
\hline Hospital & 22 & 14.6 \\
\hline Other location & 9 & 6 \\
\hline Without information & 28 & 18.8 \\
\hline
\end{tabular}


Citation: Latorre ML, Zambrano C, Moreno S (2016) Sudden Infant Death Syndrome: Forensic Autopsy Findings in Post-neonatal Deaths.

Page 3 of 5

\begin{tabular}{|l|l|l|}
\hline Activity at time of death & 39 & 26 \\
\hline Sleep or Rest & 22 & 14.6 \\
\hline Activity in home & 5 & 3.3 \\
\hline Other & 84 & 56 \\
\hline Without Information & & \\
\hline Manner of death & 72 & 48 \\
\hline Natural & 58 & 38.6 \\
\hline Under study & 8 & 5.3 \\
\hline Indeterminate & 3 & 2 \\
\hline Violent-accidental & 1 & 0.6 \\
\hline Violent-homicide & 8 & 5.3 \\
\hline Violent-indeterminate & & \\
\hline
\end{tabular}

causes were then disregarded, and with this information a first classification was done to see which cases were compatible with SIDS. 114 cases in which the parents have said they put the baby to sleep and later they found the baby cold and cyanotic were selected; some of these cases had a record with the baby having an acute respiratory infection - ARI.

The 36 cases diagnosed had a probable cause of death: 2 stillbirth, 1 miscarriage, 6 trauma cases confirmed to be nonrelated with sleeping, 27 cases of infants severely sick that were hospitalized with diagnosis like leukemia, anencephaly, imperfect osteogenesis, sepsis, severe prematurity, pneumonia, congenital heart disease, pulmonary hypertension and other congenital malformations.

Out of the 114 possible SIDS/SUDI cases, it is noted that 3 cases had a "violent and undetermined" death cause, had similarities to SIDS cases in which the parents have said they found the baby dead on the bed after it was left sleeping.

A case in which an older infant was found with her head stuck between the mattress and the crib's railings was also included as a SUDI case. Another case in which the mother was under the influence of alcohol and woke up with her daughter underneath her without vital signs was included as well as a SUDI case. Cases like the previously mentioned are commonly diagnosed as sudden unexpected infant deaths - SUDI (Table 3).

\section{Information of the autopsy report}

The summarized facts collected in the autopsy protocols of 150 cases were revised for uniformity. The 36 cases with visible death

\begin{tabular}{|c|c|c|c|c|c|}
\hline $\begin{array}{l}\text { Cause of death in SIRDEC } \\
(n=150)\end{array}$ & Number of cases & $\begin{array}{l}\text { Cause of death in autopsy } \\
(n=150)\end{array}$ & Number of cases & $\begin{array}{l}\text { SIDS compatible cases and } \\
\text { relevant history }(n=114)\end{array}$ & Number of cases \\
\hline Trauma & 8 & Stillbirth & 2 & SIDS, no history & 95 \\
\hline Suffocation & 2 & Miscarriage & 1 & SIDS with respiratory history & 9 \\
\hline Pneumonia & 22 & Trauma & 6 & $\begin{array}{l}\text { SIDS with gastrointestinal } \\
\text { history }\end{array}$ & 6 \\
\hline Congenital malformations & 7 & Malformations, or Syndromes ${ }^{*}$ & 6 & SIDS with heart defect & 2 \\
\hline Intestinal infection disease & 1 & Congenital heart defect & 3 & $\begin{array}{l}\text { SIDS with a former acute life } \\
\text { threatening event (ALTE). }\end{array}$ & 2 \\
\hline Hepatic Causes & 1 & Other diseases $^{\star *}$ & 5 & & \\
\hline Meningitis & 1 & Sepsis & 7 & & \\
\hline Influenza & 1 & Infection ${ }^{* * *}$ & 3 & & \\
\hline \multirow[t]{3}{*}{ Death In Study } & 107 & Prematurity & 1 & & \\
\hline & & Compatible with SIDS & 114 & & \\
\hline & & Hemorrhage & 2 & & \\
\hline Total & 150 & Total & 150 & Total & 114 \\
\hline
\end{tabular}

Table 3: Cause of death in SIRDEC, autopsy report and SIDS compatible cases (Source: INMLCF, SIRDEC database and autopsy report). ( ${ }^{\star}$ Anencephaly (1 case), Cayler Syndrome (1 case), Down syndrome (1 case), Prune Belly Syndrome (1 case), Osteogenesis Imperfecta ( 1 case) and "other" (1 case); ${ }^{\star \star}$ Pulmonary Hypertension ( 2 cases), Leukemia (1 case), Burn ( 1 case), Jaundice ( 1 case); ${ }^{\star * \star}$ Pneumonia ( 1 case), Meningitis (1 case), Bronchiolitis (1 case))

Among all of the possible SIDS/SUDI cases $(n=114), 38$ infants died at the place where the corpse was removed. In other 2 cases, the infant died at their kinder garden. One case doesn't have any information.
The other 73 cases, the children were taken to a hospital to which they arrived without vital signs; in some cases, it was reported that basic $\mathrm{CPR}$ was perform without success. It was mentioned that two infants 
were taken to private hospitals; the others were taken to public hospitals. Table 4 shows that 23 infants were sleeping with another person when they died and they are in the group of 95 SIDS.

\begin{tabular}{|c|c|c|c|c|c|}
\hline Variable & $\mathbf{n}$ & Variable & $\mathrm{n}$ & Variable & $\mathbf{n}$ \\
\hline \multicolumn{2}{|l|}{ Co-sleeping } & \multicolumn{2}{|l|}{ Other } & \multicolumn{2}{|l|}{ Blanket } \\
\hline $\begin{array}{l}\text { With brothers } \\
\text { or sisters }\end{array}$ & 2 & Adolescent mother & 6 & Yes & 9 \\
\hline With father & 11 & Alcoholism in mother & 2 & $\begin{array}{l}\text { Without } \\
\text { information }\end{array}$ & 141 \\
\hline $\begin{array}{l}\text { With mother or } \\
\text { father }\end{array}$ & 12 & Oxygen use & 3 & \multicolumn{2}{|l|}{ Prematurity } \\
\hline $\begin{array}{l}\text { With father and } \\
\text { brother }\end{array}$ & 2 & Vaccines & 2 & Yes & 20 \\
\hline $\begin{array}{l}\text { Without } \\
\text { information }\end{array}$ & 123 & Without Information & 135 & $\begin{array}{l}\text { Without } \\
\text { information }\end{array}$ & 130 \\
\hline
\end{tabular}

Table 4: Risk factors associated with deaths (Source: INMLCF, autopsy protocol).

Among 114 cases, 15 were deaths following an illness or symptoms of concern like respiratory (7 cases) or gastrointestinal (6 cases) related, which had received medical assistance in previous days. There was also a case in which the child had a history of heart disease and a cardiopathy was found in the autopsy of an asymptomatic boy. Two cases were compatible with suffocation. In one case, a boy was in treatment with antihistamines because he had an acute respiratory infection. Another case showed cardiopathy with signs of pulmonary hypertension.

The remaining 95 cases showed similar characteristics: the infant appeared to be healthy or presented mild signs of a common cold, and unexpectedly died while sleeping in Table 3.

There was no access to 30 complete dossiers. Among 65 files that were found, $23 \mathrm{did}$ not have the histopathology report.

Out all of the files that had the histopathology report ( $n=42), 26$ of the histopathology diagnoses were interstitial pneumonitis (chronic or acute); in two of these, aseptic meningitis and cerebral edema were found.

The histopathology diagnosis of 8 cases was acute pneumonia but only one case was given the same diagnosis as cause of death. Two cases were reported as cerebral edema. Interstitial pneumonitis and pneumonias came with edema, congestion, and signs of hypoxia in other organs. A tracheitis was also found in five infants.

\section{Discussion and Conclusion}

The SIDS diagnosis is a very complex process because it is a diagnosis by exclusion that depends upon the expertise of the pathologist, with many studies showing different opinions of what constitutes a sufficient cause of death. Even though a multidisciplinary team is imperative to close each case, a big portion of the decision of the final diagnosis depends on the autopsy. Bogota does not have a specific protocol to study SIDS and it is very difficult to find an agreed diagnosis of it. But in this study, at least 95 cases are compatible with SIDS.
Since 1969 when an International Conference at Seattle discussing the causes of infant mortality was held, a consensus accepted by the U.S. National Institutes of Health (NIH) was created. In this consensus, the Sudden Infant Death Syndrome (SIDS) was standardized for the first time as the inexplicable death of an infant by medical records and which remained without a probable cause of death after a thorough post mortem exam that included a death scene investigation $[6,9,10]$.

The infant deaths in which an evident cause was not found started to be encoded as SIDS (ICD-8 796.0, currently ICD-10 R95). However, the forensic pathologists didn't feel comfortable by using this diagnosis in cases where the major risk factors such as co-sleeping (overlaying) and the prone sleeping position (suffocation) could have been the cause. This led to the use of different terms such as borderline SIDS, unsafe sleeping conditions compatible with SIDS, secondary SIDS, and even undetermined cause of death $[6,9,10]$. In Colombia, forensic pathologist can choose between the following causes of death: natural, accidental violent, homicide-violent, suicide-violent, undetermined, and under study.

A group of expert forensic pathologists in 2004 [5] after recognizing the challenges of completing all the under study case investigations and the need to standardize concepts. To get proper classifications, they proposed that these deaths keep being labelled as SIDS unless they find a complete and sufficient cause. This death may not be explained within the limits of the investigation and knowledge. The sudden death cases with an identifiable possible cause will be labelled as SUDI and will pass through various levels of certainty classified according to the findings included on the infant's history of medical records, the death scene investigation, and the results from the post mortem study. It allows the causes of death to be classified taking into account the incomplete information, the risk factors present. These may have a major or minor impact on the result as well as taking into account that the autopsy results and the histopathology interpretation depends on the expertise of the responsible pathologist.

Within the SIRDEC's information and review of the autopsy reports, occasional inconsistencies are often found as for the infant's age. For example, SIDS has been given an upper age limit of 364 days for research purposes because of the higher likelihood of false positive SIDS over one year. These differences are even more prominent in the INMLCF and DANE data even though the death certificate is always the same.

In the INMCLF Forensic Guide, the topic of SIDS includes the complementary exams that must be performed such as histology, toxicology, biology, a full body xray and any other special study in case there is a suspicion of a hereditary disease [7], but this requirement is often not met.

According to the reviewed information collected by the INMLCF, the accurate diagnosis of SIDS is highly limited since nowadays not all the pertinent exams such as the metabolic profile, amino acid or toxicological tests, etc., used to discard other causes of death are not being performed.

In conclusion, as a result of this study it's recognized that SIDS exists in Bogota and that the current cases are not being studied with the depth and thoroughness necessary to provide an accurate diagnosis. The SIDS classification to use, depends on the possibility to find the complete information in the whole Country, not just Bogota, and the SIDS research protocol must be built by a group of Colombian and International experts from different medical disciplines. 
Citation: Latorre ML, Zambrano C, Moreno S (2016) Sudden Infant Death Syndrome: Forensic Autopsy Findings in Post-neonatal Deaths.

Page 5 of 5

There is still disagreement whether this is really a syndrome or an inexplicable entity that must be called as it is: the inexplicable and unexpected sudden death of infants. However, the diagnoses and classifications of the possible SIDS cases still have lot discrepancies. The possibility to have the most amount of information about each of the cases that get to the INMLCF, as the Inspection Act requires, increases the possibility of reconstructing the deaths scene, and will help clarify some of the causes of death.

Other countries' pathologists [11] and the experience of the pathologists of the Colombia INMLCF, all agree in finding similar classic stories in which the mother states she fed the baby at dusk, put him to sleep and, then, found him dead the next morning. Similar stories to this one were found in the files reviewed for this study whenever there was a recorded declaration from the parents [12].

While there are big conceptual discrepancies at the time of diagnosing the causes of death of an infant's inexplicable and unexpected sudden death, there is indeed a global consensus as to how to minimize them. This is by the promotion of "safe sleep" practices that must be used with every baby at their sleep hours both during night-time and daytime naps. Practices such as using the supine position for infant sleep, avoiding co-sleeping and overheating, avoiding use of alcohol when pregnant, and parental tobacco smoking, and using a list of other safe practices need to be applied to avoid more cases of SIDS in the future.

\section{Acknowledgment}

The authors thank Fundación Conocimiento (Knowledge Foundation) from Bogotá, Colombia, for their support to the realization of this research.

\section{References}

1. (2014) Centers for Disease Control and Prevention (CDC). Sudden Unexpected Infant Death and Sudden Infant Death Syndrome (Web site).

2. Krous HF, Byard RW (2001) International Standardized Autopsy Protocol for Sudden Unexpected Infant Death. Appendix 1 Sudden Infant Death Syndrome Problems, progress and possibilities. London pp: 319-333.

3. The Royal College of Pathologists and The Royal College of Paediatrics and Child Health (2004) Sudden unexpected death in infancy A multiagency protocol for care and investigation, The report of a working group convened by The Royal College of Pathologists and The Royal College of Paediatrics and Child Health pp: 1-78.

4. Fried KB, Goodwin MS, Lipsitt LP (2004) Alcohol use and Sudden Infant Death Syndrome. Developmental Review 24: 235-251.

5. Zhang K, Wang X (2013) Maternal smoking and increased risk of Sudden Infant Death Syndrome: A meta-analysis. Leg Med (Tokyo) 15: 115-121.

6. Beckwith JB (2003) Defining the Sudden Infant Death Syndrome. Arch Pediatrics Med 157: 286-290.

7. Instituto Nacional de Medicina Legal y Ciencias Forenses. Guía de procedimientos para la realización de necropsias medicolegales, segunda edición. 2004. Páginas 62-63.

8. Kinney HC, Thach BT (2009) The Sudden Infant Death Syndrome. N Engl J Med 361: 795-805.

9. Blair PS, Byard RW, Fleming PJ (2009) Proposal for an International Classification of SUDI. J of Forensic Science 1: 1-40.

10. Krous HF, Beckwith JB, Byard RW, Rognum TO, Bajanowski T, et al. (2004) Sudden Infant Death Syndrome and Unclassified Sudden Infant Deaths: A Definitional and Diagnostic Approach. Pediatrics 114: 234-238.

11. Sandomirsky Marianna, MD, FCAP, CAP Forensic Pathology Resource Committee (2012). The Past, Present, and Future of SIDS, Part I of II.

12. Barkley L (2014) Sudden Infant Death Syndrome. Medscape. 